\title{
CAPSULE COMMENTARIES \\ Capsule Commentary on Calcaterra et al., Opioid Prescribing at Hospital Discharge Contributes to Chronic Opioid Use
}

\author{
Matthew S. Subramani, MD \\ Department of Internal Medicine, Medical College of Wisconsin, Milwaukee, WI, USA.
}

J Gen Intern Med 31(5):532

DOI: $10.1007 / \mathrm{s} 11606-016-3587-4$

(c) Society of General Internal Medicine 2016

$\mathrm{C}$ alcaterra et al. ${ }^{1}$ found that among 6889 opiate-naïve patients discharged from the hospital over a 12-month period, $25 \%$ were prescribed opiates upon discharge. One year later, the odds of still being on opiates was higher among those who were prescribed opiates on discharge than those who were not (OR: 3.39, $95 \%$ CI: 2.40-4.79). Moreover, the effect among medical patients was higher than that among surgical patients, with adjusted odds ratios of 7.24 and 3.40, respectively.

Calcaterra et al. noted that inpatient physicians are less likely to be able to characterize the potential for opioid abuse than their primary care counterparts, who have more longstanding relationships with their patients. Patients discharged on opiates were more likely to be female, enrolled in discount payment plans, and to have had an admission diagnosis of chronic pain and a discharge diagnosis of acute or chronic pain, were less likely to have known substance abuse or mental health disorders, and had higher hospital readmission rates. In addition, patients discharged on opiates had higher rates of hospitalization over the 1-year follow-up. However, the more clinically significant question regarding characteristics of patients likely to be on opiates at 1 year was not addressed. The authors give an overview of who is more likely to be prescribed opioids, but do not help inpatient providers predict patients who are more likely to develop chronic opioid use. Furthermore, the study reported the frequency of specific opioids prescribed but did not comment on which drugs were most likely to be associated with chronic use.

This relationship between long-term use of opiates after being prescribed them on discharge has been seen before; inpatient providers should be aware that when they decide to discharge a patient on opiates, there is a threefold increase in the likelihood that the patient will still be taking opiates a year later. Consequently, the decision should not be made lightly. There has been a great deal of emphasis in ambulatory medicine on best practices for prescribing opiates, including assessing risk of abuse, obtaining urine drug screens, having both patient and provider sign opiate consents, and checking the state prescription drug monitoring system. Little attention has been paid to best practices for prescribing opiates during the transition from inpatient to outpatient settings. Future research could focus on this transition and on establishing best practices for opiate use in this setting.

Corresponding Author: Matthew S. Subramani, MD; Department of Internal MedicineMedical College of Wisconsin, Milwaukee, WI, USA (e-mail: msubramani@mcw.edu).

\section{Compliance with Ethical Standards:}

Conflict of Interest: This author has no conflicts of interest with the material in this article.

\section{REFERENCES}

1. Calcaterra S, et al. Opioid prescribing at hospital discharge contributes to chronic opioid use. J Gen Intern Med. 2015. doi:10.1007/s11606-0153539-4.

2. Alam A, Gomes T, Zheng H, Mamdani MM, Juurlink DN, Bell CM. Longterm analgesic use after low-risk surgery: a retrospective cohort study. Arch Intern Med. 2012;172(5):425-30. 